Article

\title{
Sol-Gel Treatments to Flame Retard PA11/Flax Composites
}

\author{
Fabienne Samyn ${ }^{1}\left[\right.$, Maxence Vandewalle ${ }^{2}$, Séverine Bellayer ${ }^{1}$ and Sophie Duquesne ${ }^{1, *} *$ \\ 1 CNRS, INRA, ENSCL, UMR 8207-UMET-Unité Matériaux et Transformations, Université de Lille, \\ F-59000 Lille, France; fabienne.samyn@ensc-lille.fr (F.S.); severine.bellayer@ensc-lille.fr (S.B.) \\ 2 CNRS, Centrale Lille, ENSCL, Université d'Artois, UMR 8181-UCCS-Unité de Catalyse et Chimie du Solide, \\ Université de Lille, F-59000 Lille, France; Maxence.Vandewalle@ensc-lille.fr \\ * Correspondence: sophie.duquesne@ensc-lille.fr; Tel.: +33-(0)320434990
}

Received: 11 April 2019; Accepted: 16 September 2019; Published: 7 October 2019

\begin{abstract}
This work investigates the efficiency of sol-gel treatments to flame retard flax fabric/PA11 composites. Different sol-gel treatments applied to the flax fabrics were prepared using TEOS in combination with phosphorus and/or nitrogen containing co-precursors (DEPTES, APTES) or additives (OP1230, OP1311). When the nitrogen and the phosphorus co-precursors were used, two coating methods were studied: a 'one-pot' route and a successive layer deposition method. For the "one-pot" method, the three precursors (TEOS, DEPTES, and APTES) were mixed together in the same solutions whereas for the different layers deposition method, the three different treatments were deposited on the fibers successively, first the TEOS, then a mix of TEOS/DEPTES, and finally a mix of TEOS/APTES. After deposition, the sol-gel coatings were characterized using scanning electron microscope, electron probe microanalyzer, and ${ }^{29} \mathrm{Si}$ and ${ }^{31} \mathrm{P}$ solid-state NMR. When only TEOS or a mix of TEOS and DEPTES is used, homogeneous coatings are obtained presenting well-condensed $\mathrm{Si}$ units (mainly $\mathrm{Q}$ units). When APTES is added, the coatings are less homogenous and agglomerates are present. A lower condensation rate of the Si network is also noticed by solid-state NMR. When additives are used in combination with TEOS, the TEOS forms a homogenous and continuous film at the surface of the fibers, but the flame retardants are not well distributed and form aggregates. The flame retardant (FR) efficiency of the different treatments on flax fabrics was evaluated using horizontal flame spread test. The following ranking of the different systems is obtained: TEOS + Additives $>$ TEOS $>$ TEOS + DEPTES $\sim$ TEOS + DEPTES + APTES $>$ multilayers. All the sol-gel coatings improve the flame retardant properties of the flax fabric, except the multilayer treatment. Based on these results, the three most efficient sol-gels were selected to prepare sol-gel-modified flax/PA11 composites. The composite modified with only TEOS showed the best FR properties. Surprisingly, the composite modified with the phosphorus-based flame retardant (AlPi) did not exhibit improved FR properties. This effect was attributed to the fact that the amount of the FR additive deposited on the fabrics was too low.
\end{abstract}

Keywords: sol-gel; flame retardancy; flax fibers; composites

\section{Introduction}

Composite materials are increasingly being used in high added value applications, such as transportation or buildings, due to their high performance/weight ratio. Among the various types of materials that have been considered, biocomposites are of prime interest as they have a low environmental impact during their use phase (in the case of transportation, a reduction in carbon dioxide emission has been noticed due to lighter vehicles) and their manufacturing phase (natural 
resources are used to prepare them). Less reliance on foreign oil resources is an additional advantage of such biobased materials.

Considering such biobased composites, either the matrix and/or the reinforcement can be obtained from the biomass. However, regarding the choice of the matrix, the use of thermoplastic matrices leading to a final material presenting the ability of being recycled at the end of its life is, thus, favored when sustainability is considered. Among the different matrices available on the market, polyamide- 11 (PA11) has been known for several decades. It has however only recently been drawing the attention of researchers due to the interest presented by biobased polymers. Obtained from castor oil, PA11 is an engineering polymer presenting high strength and high chemical stability, while also having an excellent yield.

Regarding the fibers, society also requires the use of more renewable resources. In this context, the substitution of glass fibers in composite materials by more environmentally friendly products, such as natural fibers, is more and more attractive to the scientific community. Flax fibers appear to be a promising reinforcement, due to their high mechanical properties. Indeed, flax fibers present very good mechanical properties and are as tough as conventional glass fibers.

Flax/PA11 composites have been poorly reported in the literature [1-4], and interest in them is relatively recent. Those papers have mainly been focusing on the optimization of processing conditions of PA11 in order to optimize the mechanical properties of such composites. Indeed, since PA11 has a high melting temperature [5], it could limit its application for natural fiber-reinforced composites (NFRC). Those studies demonstrate that high mechanical properties can be obtained when the manufacturing process of PA11/flax fiber composites is carefully selected, confirming the potential of these types of materials for various industrial applications. However, until now, no paper has investigated the behavior of such materials when exposed to fire, which is of prime interest when applications such as transportation or building are considered.

The flame retardancy of composites can be obtained through different aspects. FR properties can be either provided by the material itself, the reinforcement fibers or fabric, or by using a protective coating [6-8]. However, it must to be taken into account that continuous fiber-reinforced composites are prepared using specific methods that are not always compatible with conventional methods used to flame retard short fibers/polymer composites. Indeed, fire retardant agents are usually added to matrices during the extrusion process, which cannot always be considered for composite preparation. The most used strategy to flame retard a composite consists of modifying the resin by the incorporation of flame retardant additives; various types of flame retardants have already been studied, such as halogenated or phosphorus compounds, melamine derivatives, metal hydroxides, and nano- or microparticles $[9,10]$. However, this approach is limited by the process since the fabric of dry fibers could act as a filter for FR additives during processing. Therefore, the FR homogeneity inside the composite would be affected. Moreover, the mechanical properties of the final composites could also be affected. Another approach consists of protecting the composites with a coating after its processing. This technique avoids the previously described problems, but introduces additional processing steps during the manufacturing of the composite. Moreover, adhesion and durability problems can also appear. The coating approach that has been used in this study consists of a treatment of the fibers themselves. The fabrics and the plies are used as a carrier to provide FR properties to the composite. Such an approach has recently been reported in the literature $[8,11-13]$ and has shown interesting results.

In this context, the objective of this paper is to develop flame retardant Flax/PA11 composites. The flax fibers were treated to improve their fire-retardant properties. The literature concerning FR treatments of cellulosic fibers is quite extensive [14-16], and recent research has mainly been focusing on the use of sol-gel processing [17-21]. It is the reason why this method was selected in our study. In the first part of this study, flax fabric was treated using different sol-gel treatments and was fully characterized to identify the coating morphology. The influence of the processing parameters of the sol-gel, as well as of the formulation, was investigated. In the second part, the fire-retardant 
performance of the functionalized composites prepared using the treated plies are compared and discussed to link the performances of the composites to their formulation.

\section{Materials and Methods}

\subsection{Materials}

Films of PA11 (thickness of $200 \mu \mathrm{m})$ and $2 / 2$ twill-weaved woven flax fabric $\left(350 \mathrm{~g} / \mathrm{m}^{2}\right)$ have kindly been provided respectively by Arkema (Colombes, France) and Depestele Group (Burguebus, France). Three precursors (Figure 1) were used in the sol-gel treatments: tetraethoxysilane (TEOS), diethylphosphatoethyltriethoxysilane (DEPTES), and (3-aminopropyl) triethoxysilane (APTES). DEPTES (purity 95\%) has been purchased from ABCR. TEOS (purity 98\%), $\mathrm{HCl}(0.1 \mathrm{M})$, APTES, and ethanol were obtained from Sigma-Aldrich and used as received without any purification. Exolit OP1230 and OP1311, provided by Clariant, correspond, respectively, to aluminum phosphinate (AlPi) and to a mixture of aluminum phosphinate and melamine polyphosphate (AlPiMPP). They have been used in the sol formulation as flame retardant additives.

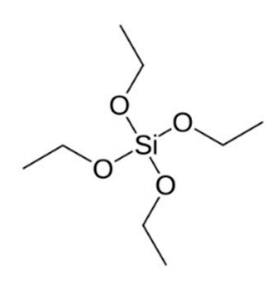

T

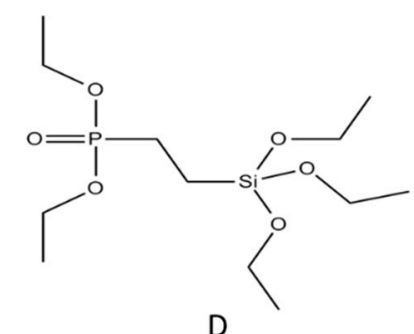

D

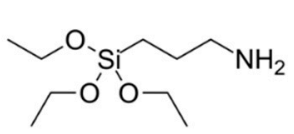

A

Figure 1. Silane precursors used in the study $(\mathrm{T}=\mathrm{TEOS}, \mathrm{D}=\mathrm{DEPTES}$, and $\mathrm{A}=\mathrm{APTES})$.

\subsection{Sol-Gel Treatments of the Fibers}

The different sol-gel formulations tested in this work are detailed in Table 1. The precursor(s) TEOS, APTES, and DEPTES-alone or in mixtures, with or without FR additives, are hydrolyzed using $\mathrm{HCl}(0.1 \mathrm{M})$ in a mix of ethanol and deionized water under vigorous stirring for $4 \mathrm{~h}$ at room temperature. All solutions coded A were prepared according to the procedure described by Alongi et al. [21]. The solution labeled B were prepared with the same precursor/ $\mathrm{HCl} / \mathrm{ethanol}$ ratios as in the A solutions, but with a significantly lower water content in order to increase the concentration of precursors in the media. The reduction of the $\mathrm{H}_{2} \mathrm{O}$ /precursor ratio can greatly influence the kinetics of formation of the coatings as it will reduce the hydrolysis step and delay the gelling time [22]. Such modification of the reaction kinetics could significantly affect the morphology of the coatings and, thus, the performance of the resulting composites. The TEOS/DEPTES ratio has been chosen as 70/30, accordingly to the literature. Indeed, Cardenas et al. [23] stated that it is the optimal ratio to build a good 3D network. As a FR synergistic effect is usually observed when both phosphorus and nitrogen are combined $[19,24,25]$, a combination of DEPTES and APTES was also considered in the present work. In that case, two processes are considered: a one-pot solution (A-TDA) or a successive layer deposition method (A-T/TD/TA, TEOS is first deposited (layer 1, L1) followed by DEPTES (layer 2, L2) and then APTES (layer 3, L3)).

The $16 \times 6 \mathrm{~cm}^{2}$ and $12 \times 12 \mathrm{~cm}^{2}$ samples were treated using $100 \mathrm{~mL}$ and $150 \mathrm{~mL}$ of the different sol-gel solutions, respectively, presented in Table 1. The samples were immersed in the solution for 1 $\min 30 \mathrm{~s}$. The excess of solution was removed using a roll padder by applying a pressure of $0.2 \mathrm{MPa}$ in one pass. The sample was then dried overnight in a ventilated oven at $80^{\circ} \mathrm{C}$ and kept one days at room temperature before analysis. The treated plies were then stored in a desiccator. The typical add-on yield for such treatments is lower than 2 wt.- $\%$ when no FR additive is used and lower than 5 wt.-\% when FR additives are added to the sol-gel. 
Table 1. Sol-gel compositions (T: TEOS; D: DEPTES; A: APTES).

\begin{tabular}{|c|c|c|c|c|c|c|c|c|}
\hline Code & $\begin{array}{c}\text { Precursor } \\
\text { Ratio (wt.\%) }\end{array}$ & $\mathrm{T}(\mathrm{mL})$ & $\mathrm{D}(\mathrm{mL})$ & $A(m L)$ & Additive (g) & $\begin{array}{l}\mathrm{HCl} \\
(\mathrm{mL})\end{array}$ & $\begin{array}{c}\text { Ethanol } \\
(\mathrm{mL})\end{array}$ & $\begin{array}{l}\text { Water } \\
(\mathrm{mL})\end{array}$ \\
\hline A-T & 100 & 6.84 & & & & 8 & 5 & 80.16 \\
\hline B-T & 100 & 17.25 & & & & 20.17 & 12.61 & 50 \\
\hline A-TD & $70 / 30$ & 4.79 & 2.05 & & & 8 & 5 & 80.16 \\
\hline $\mathrm{B}-\mathrm{TD}$ * & $70 / 30$ & 12.07 & 5.17 & & & 20.17 & 12.61 & 50 \\
\hline A-TDA & $55 / 38 / 7$ & 3.76 & 2.62 & 0.46 & & 8 & 5 & 80.16 \\
\hline \multicolumn{9}{|l|}{$\mathrm{A}-\mathrm{T} / \mathrm{TD} / \mathrm{TA}$} \\
\hline L1:T & 100 & 6.84 & & & & 8 & 5 & 80.16 \\
\hline L2:TD & $70 / 30$ & 4.79 & 2.05 & & & 8 & 5 & 80.16 \\
\hline L3:TA & $85 / 15$ & 5.81 & & 1.03 & & 8 & 5 & 80.16 \\
\hline A-TAlPi & 100 & 6.84 & & & 0.8885 & 8 & 5 & 80.16 \\
\hline B-TAlPi * & 100 & 17.44 & & & 2.2407 & 20.17 & 12.61 & 50 \\
\hline A-TAlPiMPP & 100 & 6.84 & & & 1.044 & 8 & 5 & 80.16 \\
\hline
\end{tabular}

\subsection{Composite Preparation}

Four-ply composites were prepared by a method combining film stacking and vacuum bagging to ensure a good impregnation of the fabric by the matrix. The stacking was performed in a steel mold using five layers of PA11 (one layer corresponding to a $200 \mu \mathrm{m}$ thick film) and four flax fabrics that were disposed alternatively. A bag (Nylon $66, \mathrm{Tf}=232{ }^{\circ} \mathrm{C}, 50 \mu \mathrm{m}$ thick, $17.4 \mathrm{~m}^{2} / \mathrm{kg}$, from Cytec) is then sealed around the mold, and a vacuum is maintained throughout the whole process using a vacuum pump. The assembly was pressed (Servitec Polystat 200 press, Servitec Maschinenservice $\mathrm{GmbH}$, Wustermark, Germany) for consolidation purpose for $6 \mathrm{~min}$ at $195^{\circ} \mathrm{C}$ under 20 bar pressure. This temperature was selected to avoid fiber degradation, and to allow the melting of PA11, which typically occurs between 180 and $190{ }^{\circ} \mathrm{C}$. The vacuum is maintained during the whole process and removes when after cooling the mold at ambient temperature. The fiber content in the composite was estimated to be $(55 \pm 5) \mathrm{wt}$.- $\%$ (the amount of sol-gel deposited is neglected in the calculation).

\subsection{Characterization}

\subsubsection{Electronic Microscopy Analyses}

The morphologies of the virgin and of the different coated flax fibers were observed using a scanning electron microscope (SEM), Hitachi 3400 Ntype II, at an accelerating voltage of $15 \mathrm{kV}$. Images were taken at various magnifications to ensure representative imaging of the samples. Using an energy-dispersive X-ray spectrometer coupled to the SEM, the presence of silicon, phosphorous, and nitrogen has been verified. Additionally, electron probe microanalyses (EPMA) were carried out using a CAMECA SX100 instrument (Cameca, Paris, France) at $15 \mathrm{kV}$ and $40 \mathrm{nA}$. The fibers samples were carbon coated with a Bal-Tec SCD005 sputter coater (BAL-TEC GmbH, Schalksmühle, Germany) prior to analysis. Phosphorus, silicon, and nitrogen were analyzed, and X-ray mappings were built. The concentration of the analyzed element was shown on colored coded images (from red, which is used for a high concentration, to black color that is used for a low concentration).

\subsubsection{Solid-State NMR Characterizations}

The condensation level of the sol-gel obtained on the fibers has been investigated using ${ }^{29} \mathrm{Si}$ solid-state NMR. The measurements have been performed on a Bruker Avance II 400 at $19.6 \mathrm{MHz}$ using a $7 \mathrm{~mm}$ probe, with ${ }^{1} \mathrm{H}-{ }^{29} \mathrm{Si}$ cross-polarization (CP) and magic angle spinning (MAS) at a speed of $5 \mathrm{kHz}$. The relaxation time was fixed at $3 \mathrm{~s}$. The spectra were acquired using 1024 scans. The reference used was TMS.

${ }^{31} \mathrm{P}$ NMR analyses of the samples containing phosphorous either in the co-precursor or in the additives have also been performed to observe potential modifications of the phosphorous environment 
resulting from interactions/degradation during the treatments. The measurements have been performed on a Bruker Avance II 400 at $40.5 \mathrm{MHz}$ using a $4 \mathrm{~mm}$ probe, with dipolar decoupling (DD) and magic angle spinning (MAS) at a speed of $12.5 \mathrm{kHz}$. The delay time between two impulses was fixed at $60 \mathrm{~s}$. The spectra were acquired using 16 scans. The reference used was $85 \% \mathrm{H}_{3} \mathrm{PO}_{4}$ in aqueous solution.

\subsubsection{Fire Tests}

The flame spread rates of treated fabrics were measured using a horizontal flame spread test that has been developed in our laboratory. Even if the test does not correspond to any standard, it is similar to the FMVSS 302 fire test. A sample, $150 \mathrm{~mm} \times 50 \mathrm{~mm}$, is fixed into a sample holder and a burner flame is applied for $10 \mathrm{~s}$ on the sample. The flame spread rate is then measured.

A mass loss calorimeter (MLC) supplied by FTT (Fire Testing Technology, East Grinstead, USA) was used to perform the measurements on composite samples following the procedure defined in ISO 13927. In this equipment, the heat release rate (HRR) values are obtained using a thermopile placed in the chimney. Our procedure involved exposing specimens measuring $100 \times 100 \times 2 \mathrm{~mm}^{3}$ in horizontal orientation. An external heat flux corresponding to common heat flux in mild fire scenario of $35 \mathrm{~kW} / \mathrm{m}^{2}$ was used to perform the experiments. Different parameters were determined from these measurements: the peak of heat release rate (pHRR), the time to ignition (ti), the total heat release (THR) corresponding to the area under the HRR curve (here measured at $600 \mathrm{~s}$ ), as well as the residual weight (RW; values in the table are taken at $600 \mathrm{~s}$ ). Three specimens were tested for each formulations and standard deviation is lower than $10 \%$. Average data are presented for $\mathrm{pHRR}$ and THR; however, for curves, only the curve presenting the highest pHRR is presented.

\section{Results and Discussion}

\subsection{Characterization of the Fabrics}

\subsubsection{SEM Characterization}

Three precursors were considered in this study ( $T, D$, and $A)$ either alone or in combination with flame retardant additives, and two processing parameters (A and B) and two deposition methods were used when the combinations of phosphorus- and nitrogen-containing precursors are considered ("one-pot" solution and successive layers deposition).

Scanning electron microscopy coupled with EDX analyses was first carried out to verify the quality and homogeneity of the deposited coatings. The images of A-T, A-TD, and A-TDA are presented in Figure 2. The numbered dots indicate the location of the chemical analyses performed. All the data are given in the Supplementary Materials and only selected ones are included in the paper to justify the conclusions.
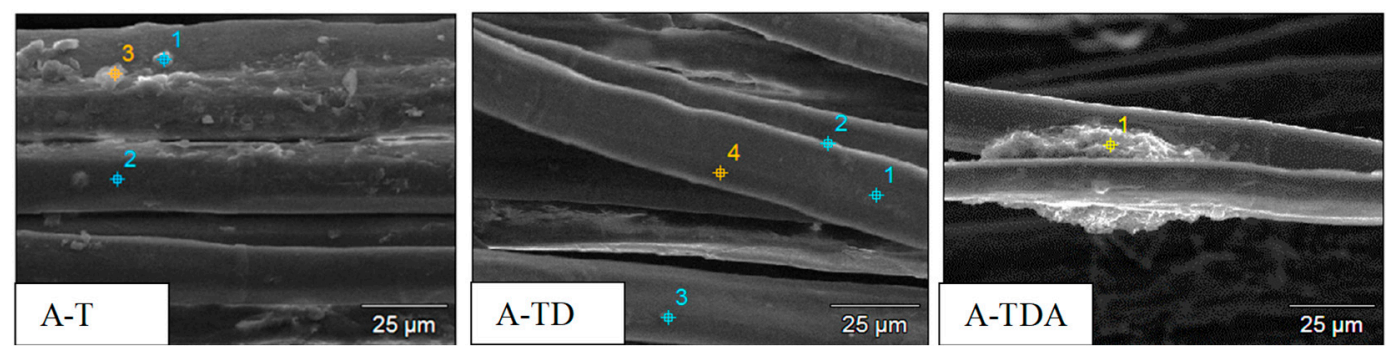

Figure 2. SEM images of treated fibers A-T, A-TD, and A-TDA $(\times 1000)$ using the processing parameters A.

From those images, it can be concluded that depending on the precursor(s), the deposit presents different morphologies. Indeed, a smooth homogeneous coating is observed on the fibers in the case of A-TD treatment, whereas for A-T- and A-TDA-treated samples, aggregates of various sizes are detected on the fibers. 
For A-T, silicon is present on the fibers as well as in the aggregates (Figure 3a,b), demonstrating that both a thin film and particles or aggregates are formed at the surface of the fibers. In the case of A-T, the size of those aggregates is relatively small (between 1 to $5 \mu \mathrm{m}$ ).

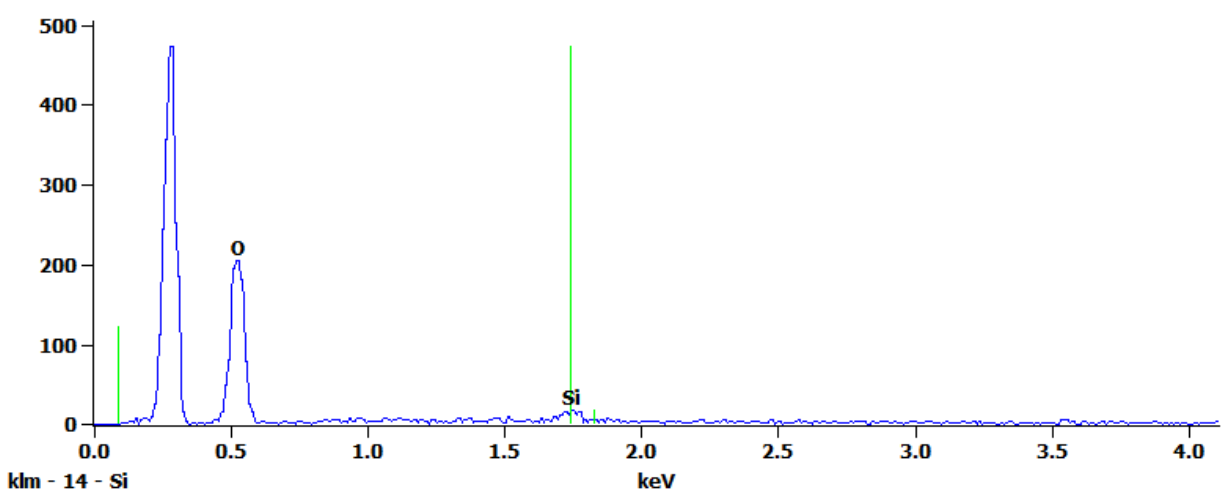

(a)

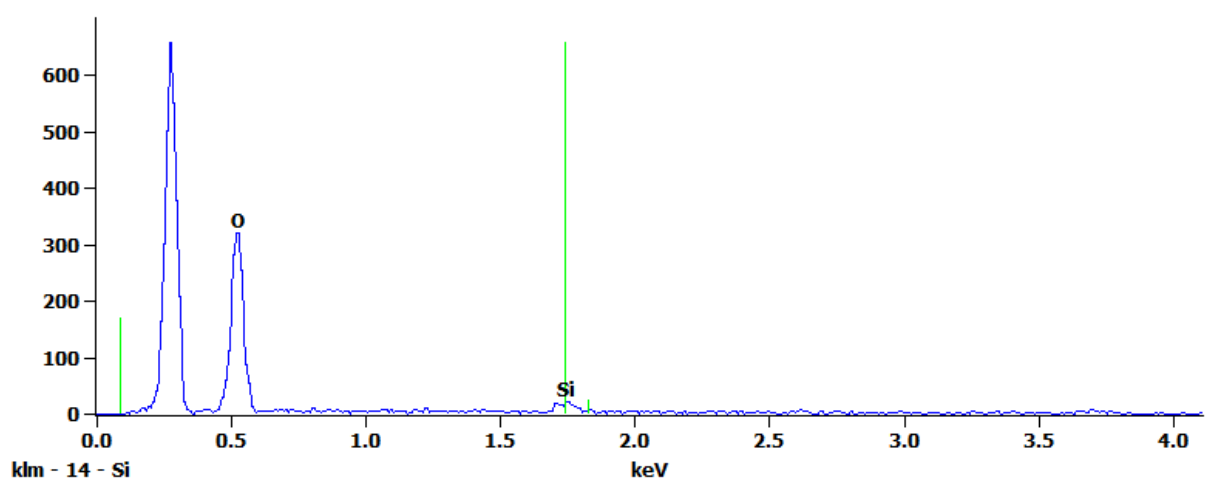

(b)

Figure 3. Energy-dispersive X-ray spectroscopy (EDX) analyses performed on the surface of the A-T fiber ((a), Point 2 of Figure 2) and on an aggregate ((b), Point 3 of Figure 2).

Considering A-TD, the behavior is different as no aggregates were observed. Micrographs were obtained at lower magnification (Figure 4) confirming this result. On the other hand, the EDX analyses (results shown as Supplementary Materials) do not permit the confirmation of the presence of phosphorus on the surface of the fibers. At this stage of the study, two hypotheses can be drawn. Either DEPTES does not condense with TEOS and remains in solution or the quantity of phosphorus is too low to be detected using SEM-EDX. Thus, to go further, EPMA analyses were carried out. EPMA has a higher detection resolution than EDX [26] and can thus provide more detailed X ray elemental mappings. The P and Si mappings of the A-TD fabric are presented in Figure 5. They confirm the presence of $\mathrm{P}$ on the surface of the flax fibers and show that $\mathrm{Si}$ and $\mathrm{P}$ are evenly distributed on the fibers. Therefore, it is possible to conclude that the presence of DEPTES affects the kinetics of condensation, since in that case, no aggregate is formed. This effect has already been reported by Cardenas et al. [23].

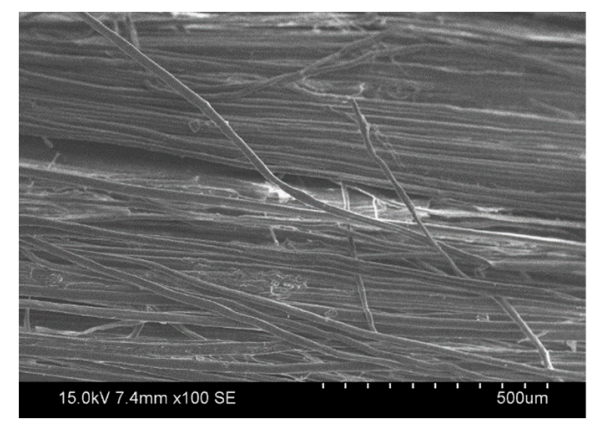

Figure 4. SEM images of A-DT at lower magnification $(\times 100)$. 

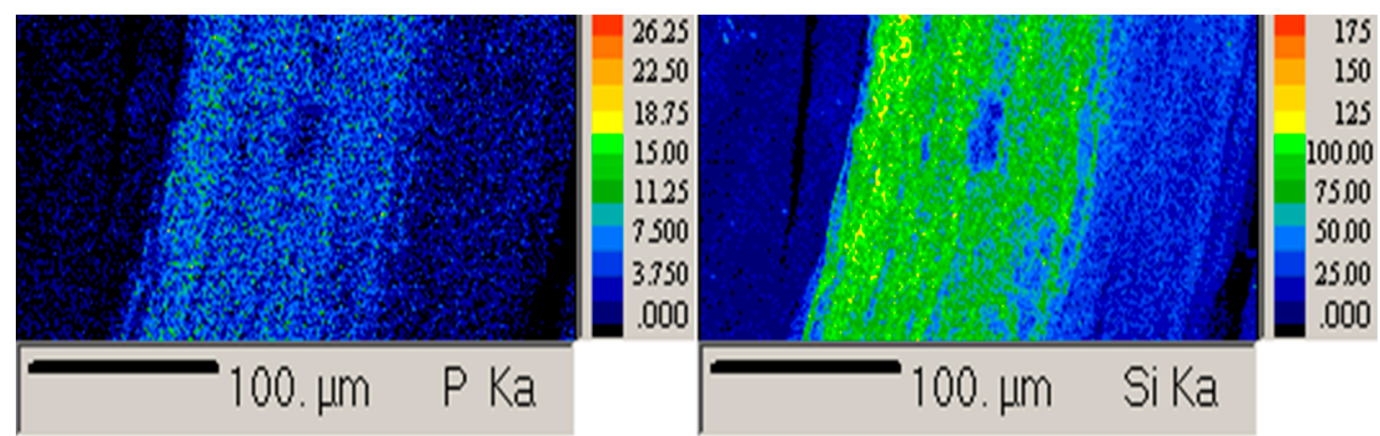

Figure 5. Wavelength-Dispersive X-Ray Spectroscopy (WDS) cartographies of phosphorus and silicon in A-TD.

Finally, in the case of A-TDA, large aggregates are observed at $\sim 10-20 \mu \mathrm{m}$. EDX analyses (results shown as Supplementary Materials) confirm that these aggregates contain $\mathrm{Si}, \mathrm{P}$, and $\mathrm{N}$, demonstrating that the three precursors are involved in the formation of the coating. Residual chlorine is also detected. EPMA was also carried out (Figure 6) on the A-TDA sample and confirmed the presence of Si, P, and N on the surface of the fibers, demonstrating that the condensation of TEOS, APTES, and DEPTES has occurred. The EPMA images are color coded and clearly show the deposition of a coating at the surface of the whole fabric (blue and green part for $\mathrm{Si}$ ); however, aggregates are noticeable (red area for $\mathrm{Si}$ ).
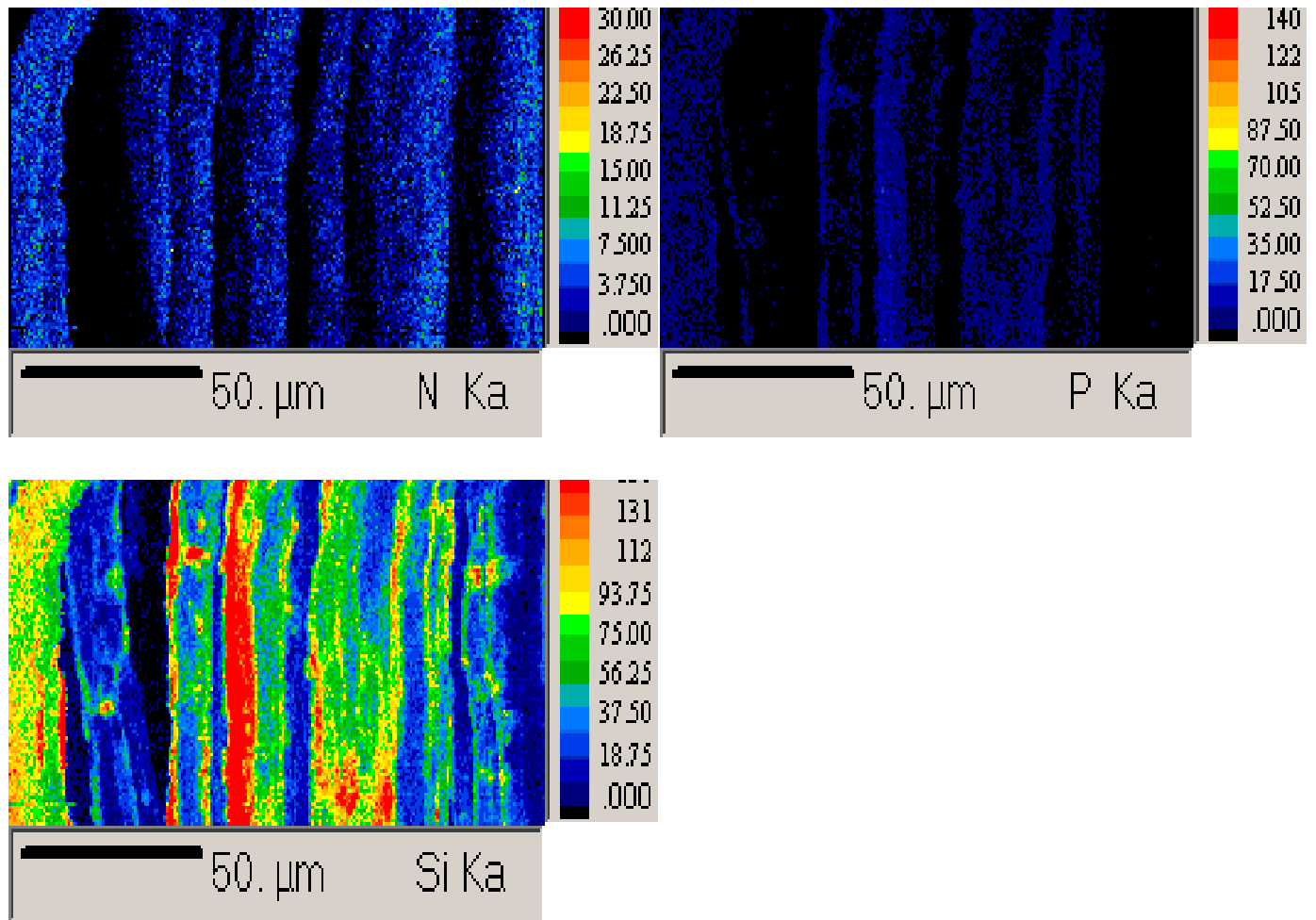

Figure 6. N, P, and Si elemental mappings of the samples, A-TDA.

The influence of the concentration of precursors was also investigated (processing method B) in the cases of T and DT. The results are presented in Figure 7. Elemental microanalysis confirmed that silicon and phosphorus (in the case of B-TD) are present on the surface of the fibers as well as in the aggregates. The results are slightly different than in processing method A. Indeed, fewer aggregates are observable for the B-T sample, whereas more aggregates are observable for B-TD. Consequently, the modification of the concentration of precursor affects the kinetics of the sol-gel coating formation. 


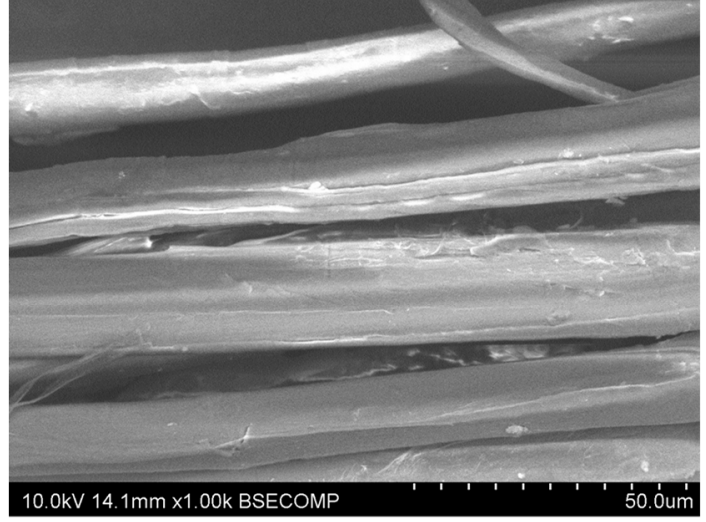

(a)

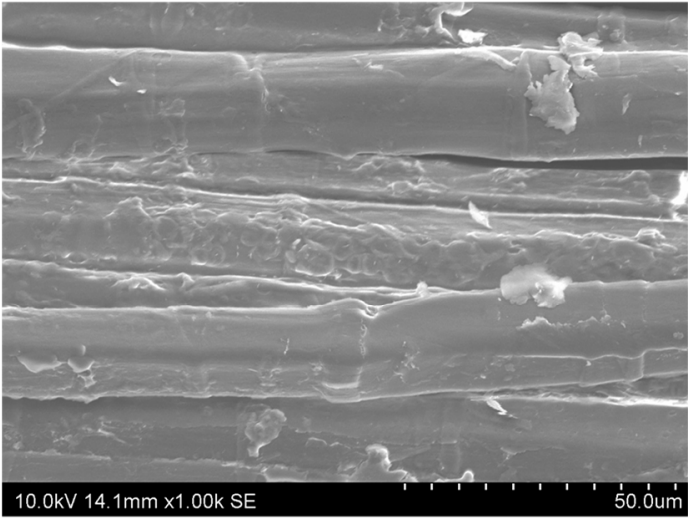

(b)

Figure 7. SEM images pictures of treated fibers B-T (a) and B-TD (b) $(\times 1000)$.

To study the effect of the deposition process on the homogeneity of the coating, the results obtained with the "one-pot" method were compared with those obtained with the successive layers deposition method. Results show that the successive layer deposition method leads to different coating structures (Figure 8). Indeed, in the case of the "one-pot" process (A-TDA), large aggregates are obtained, as previously discussed; whereas, in the case of multilayers (A-T/TD/TA), the coatings are more homogeneous.
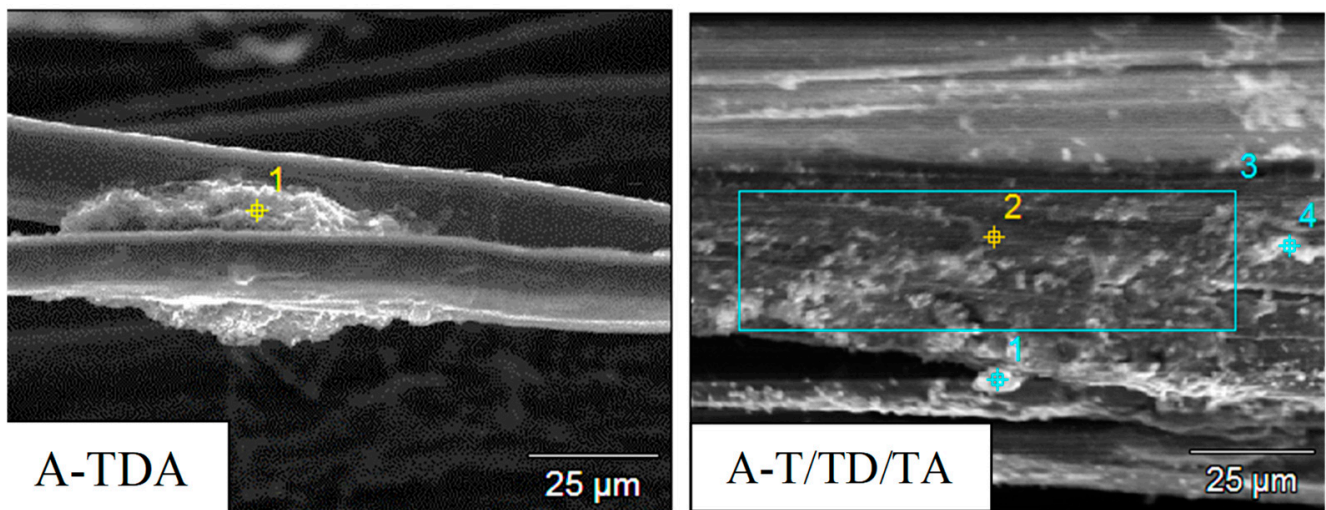

Figure 8. SEM images pictures of treated fibers $(\times 1000)$.

To further investigate and study the distribution of phosphorus and silicon on the surface of the treated fibers, EPMA analyses were carried out (Figure 9). The multilayer samples showed a higher concentrations of silicon on the top of the fibers (more concentrated areas), confirming the formation of aggregates. On the other hand, as expected no phosphorus was observed on the surface, since the DEPTES is present only in the middle layer, confirming the efficiency of the processing method.

Finally, the SEM images of the flax fibers treated with the solution containing FR additives are presented in Figure 10. Si is present on the fibers and on the aggregates and the silicon network resulting from the condensation of TEOS traps the FR particles (presence of $\mathrm{Al}$ and $\mathrm{P}$ on the aggregates) (the EDX analyses are shown in the Supplementary Materials). In these cases, the addition of FR additives does not seem to modify the condensation of TEOS. 

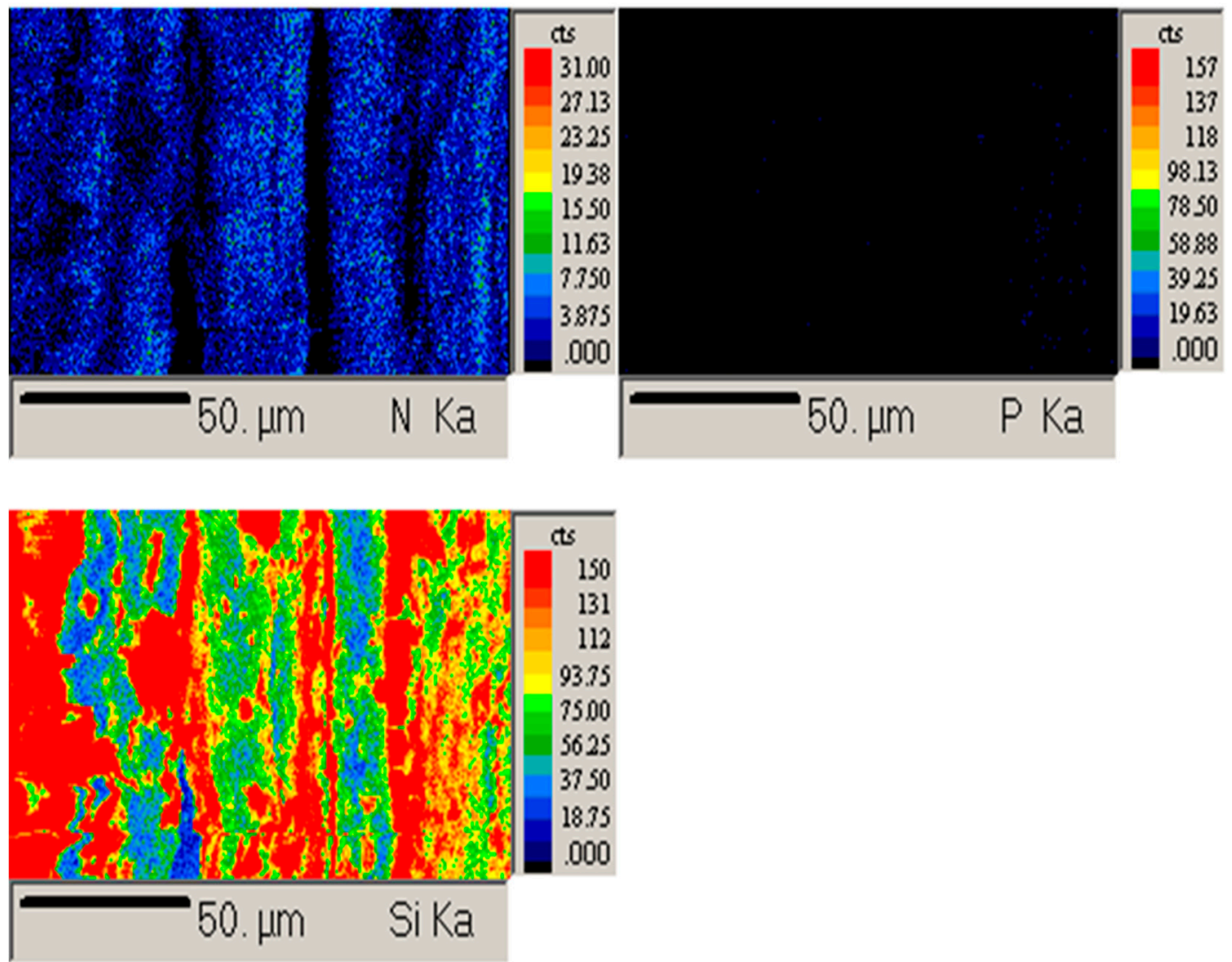

Figure 9. N, P, and Si elemental mappings of the samples, A-T/TD/TA.
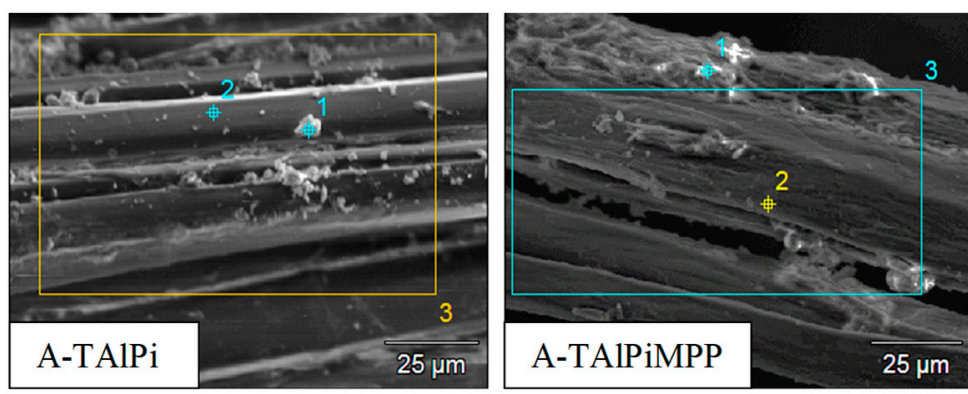

Figure 10. SEM pictures of treated fibers $(\times 1000)$.

In conclusion, those results show that the sol-gel treatments lead to two types of structures that may appear simultaneously: a thin sol-gel coating on the surface of the fiber and aggregates of various sizes. They also permit us to conclude that the presence of DEPTES in the sol-gel (either with TEOS or with both TEOS and APTES) modifies the structure of the coatings when compared to pure TEOS, and thus a modification of the condensation process is possible.

\subsubsection{Solid-State NMR}

To investigate the level of condensation of the sol-gel, ${ }^{29} \mathrm{Si}$ NMR was used. At the molecular level, solid-state ${ }^{29} \mathrm{Si}$ NMR allows the observation of connectivity and degree of condensation in solid siliceous materials. The silicone structure can be distinguished according to bridging oxygen number per silicone-oxygen tetrahedron. $\mathrm{Q}_{0}, \mathrm{Q}_{1}, \mathrm{Q}_{2}, \mathrm{Q}_{3}$, and $\mathrm{Q}_{4}$ notations used in ${ }^{29} \mathrm{Si} \mathrm{NMR}$ spectroscopy are used for $\mathrm{Si}$ with four oxygens attached; the numbers $0-4$ denote the number of ' $\mathrm{Si}$ ' units attached through the oxygen to an individual silicon atom. Similarly, $\mathrm{T}_{0}, \mathrm{~T}_{1}, \mathrm{~T}_{2}$, and $\mathrm{T}_{3}$ are used for $\mathrm{Si}$ with 
three oxygens attached; the numbers 0-3 denote the number of ' $\mathrm{Si}$ ' units attached through the oxygen to an individual silicon atom (see Supplementary Materials for further details) [27].

The spectra obtained for the treated fibers are presented on Figure 11 and the attributions are detailed in Table 2. The higher the chemical shift, the more condensed the gel. In this work, T and Q units are observed.

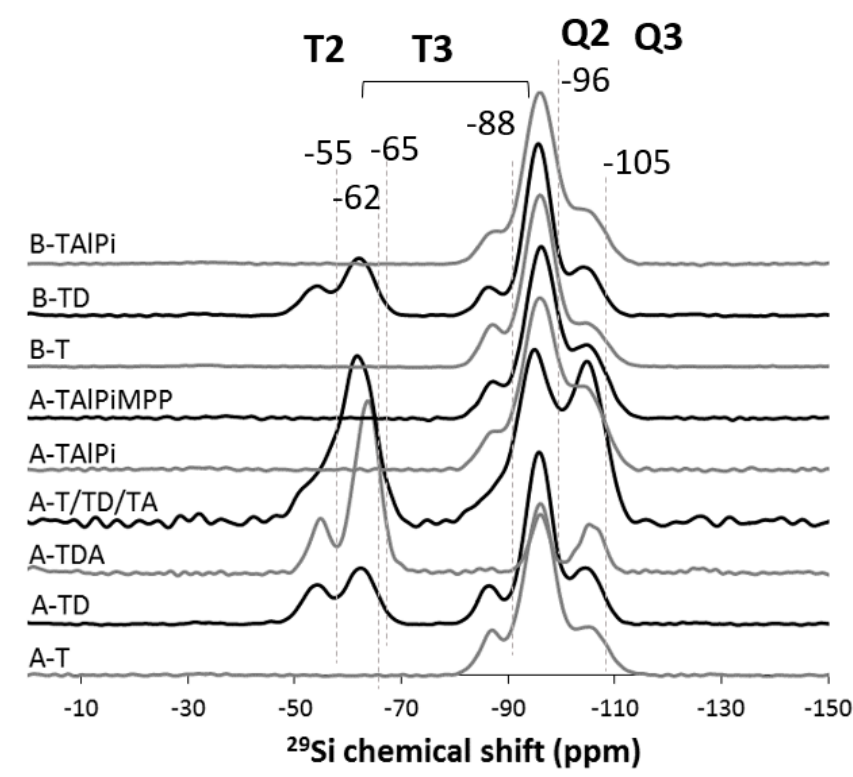

Figure 11. ${ }^{29} \mathrm{Si}$ NMR spectra of the treated fibers (normalized intensity).

Table 2. Attribution of $\mathrm{NMR}^{29} \mathrm{Si}$ and ${ }^{31} \mathrm{P}$ signals.

\begin{tabular}{ccccc}
\hline${ }^{29} \mathbf{S i}(\mathbf{p p m})$ & Assignment & Formula & ${ }^{31} \mathbf{P}(\mathbf{p p m})$ & Assignment \\
\hline-55 & $\mathrm{~T} 2$ & $\mathrm{R}-\mathrm{Si}(\mathrm{OH})(\mathrm{OSi}-)_{2}$ & 63 & \\
-62 & $\mathrm{~T} 3$ & $\mathrm{R}-\mathrm{Si}(\mathrm{OSi}-)_{3}$ & 54 & Not attributed yet \\
-65 & $\mathrm{~T} 3$ & $\mathrm{R}-\mathrm{Si}(\mathrm{OSi}-)_{3}$ & 51 & \\
\hline-88 & $\mathrm{~T} 3$ & $\mathrm{H}-\mathrm{Si}(\mathrm{OSi}-)_{3}$ & $43-41$ & AlPi \\
\hline-96 & $\mathrm{Q} 3$ & $\mathrm{Si}(\mathrm{OH})(\mathrm{OSi}-)_{3}$ & 34 & DEPTES \\
-105 & $\mathrm{Q} 4$ & $\mathrm{Si}(\mathrm{OSi}-)_{4}$ & 33 & \\
\hline
\end{tabular}

When TEOS is used alone in the sol-gel preparation, the condensation level is higher than when DEPTES or a combination of DEPTES/APTES is used. In the first case, only $\mathrm{Q}_{2}$ and $\mathrm{Q}_{3}$ units are observed, whereas $T_{2}$ and $T_{3}$ are detected with the co-precursors. It can be noticed that when the mixture between APTES and DEPTES is used, the degree of condensation is very low since the intensity of the peaks attributed to $T$ units is very intense compared to those of $Q$ units. These results also show that when FR additives are added in the solution, the condensation of the TEOS is not affected and the $Q$ structures are still obtained.

The modification of the protocol of preparation of the sol-gel consisting of an increase of the concentration of the precursors does not modify the condensation process. Indeed, whatever the formulation, the spectra obtained for A and B protocols are similar. SEM images showed some differences when comparing A-T and A-TD with B-T and B-TD samples. Those differences are attributed to the kinetics of formation of the coatings but not to their chemical structure.

Finally, when the "one-pot" method and the "layered" preparation method are compared, the condensation rate is higher for the second protocol. This result is consistent since in this case, TEOS is the first monomer to condense on the fiber surfaces, leading to the formation of more $Q$ units. It was previously demonstrated that a higher condensation is obtained when TEOS is used alone. 
The ${ }^{31} \mathrm{P}$ NMR spectra of the samples treated with DEPTES or with the FR additives are presented in Figure 12.

\section{1}

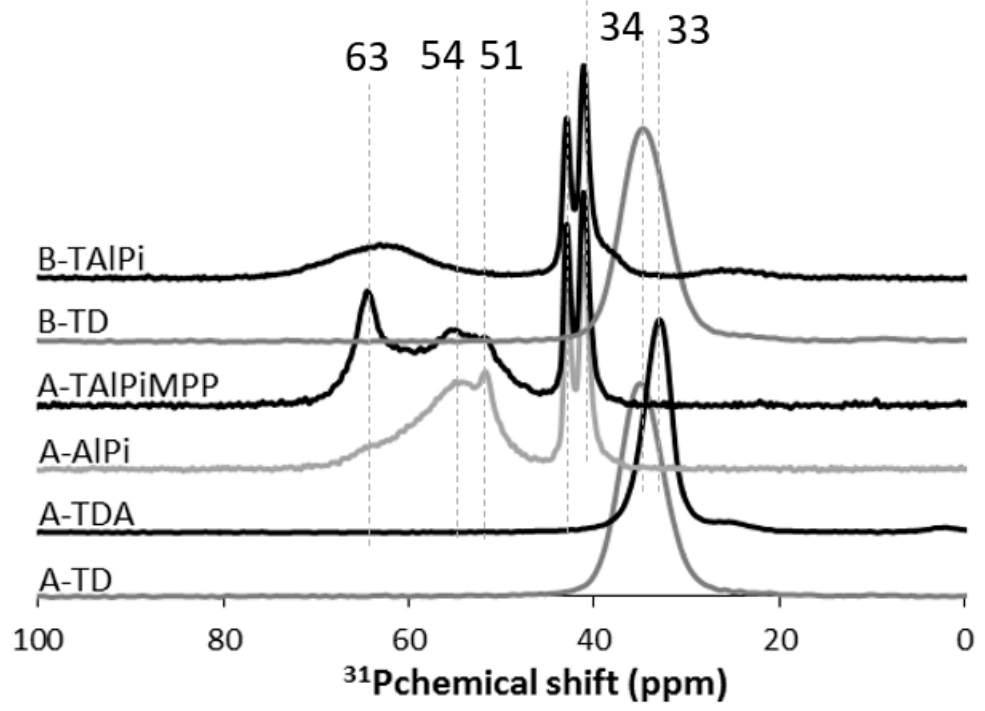

Figure 12. ${ }^{31} \mathrm{P}$ NMR spectra of the treated fibers (normalized intensity).

DEPTES exhibits a broad and a split signal between 32 and $33.5 \mathrm{ppm}$ [28]. For the samples A-TD and B-TD, a peak at 34 ppm is observed, which can thus be attributed to DEPTES. For A-TDA, the peak is shifted down to $33 \mathrm{ppm}$, but it is still considered as the DEPTES signal.

For A-TAlPi and A-TAlPiMPP, the doublet observed between 41 and $43 \mathrm{ppm}$ can be attributed to aluminum phosphinate. The other signals observed on the spectra at 51,54, and $63 \mathrm{ppm}$ are attributed to the degradation of phosphinates in acidic solution. However, these signals have not been precisely attributed yet.

The fibers have been treated and the coatings were characterized. Thus, the next step is now to measure the flame retardant properties of the treated fabrics.

\subsection{Fire Performance of the FR Fabrics}

The horizontal flame spread rate has been measured for all the coated samples (Figure 13). All the samples were dried prior to the test. The dry flax fabric behavior was used as the reference to evaluate the efficiency of the different coatings. All sol-gel coatings decreased the flame spread rate along the fabrics, except the multilayer treatment, in which the flame spread rate remains similar to the reference $(1.33 \mathrm{~mm} / \mathrm{s})$. The sol-gel containing only TEOS appears to be more efficient that the sol-gel containing co-precursors. However, the best performances were obtained with the sol-gel containing the FR additives. It was observed that the increase in precursor concentration leads systematically to a drop of the flame spread rate. The best performance is obtained for the sample B-TAlPi. For this sample, the self-extinguishment was observed twice on the three performed experiments. The lack of homogeneity of the coating at the surface of the fabrics (agglomerates were observed) obtained for this sample can explain the lack of repeatability of the flame spread rate test. 


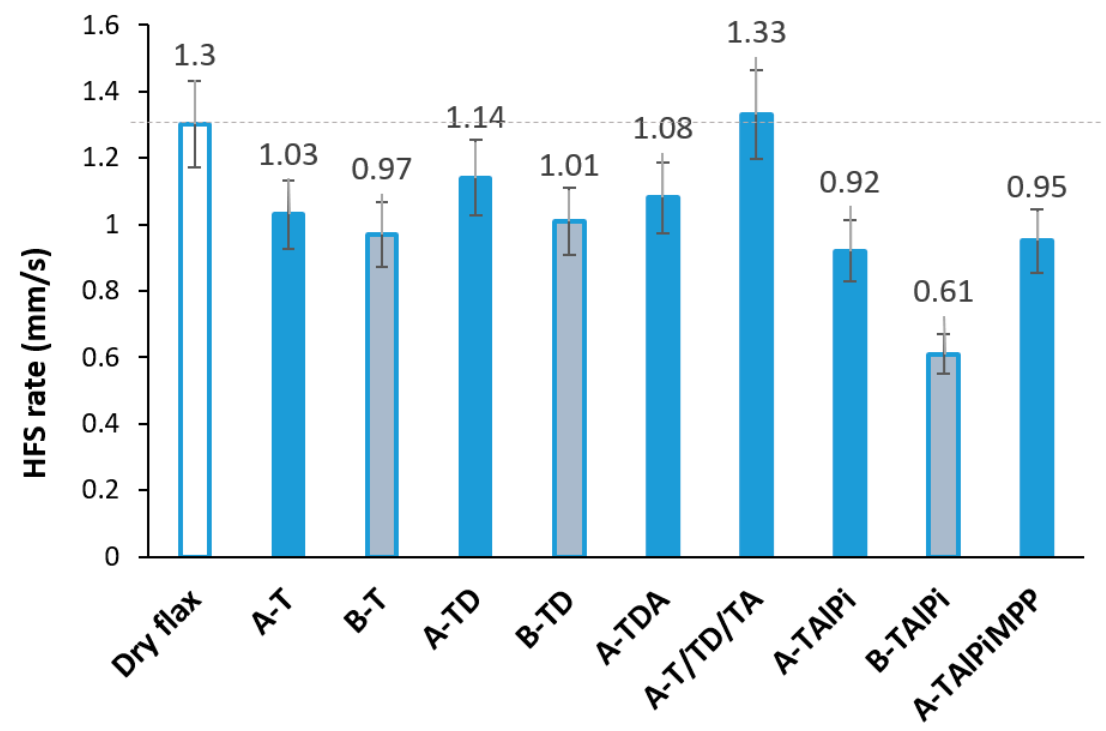

Figure 13. Horizontal flame spread (HFS) rate of the different sol-gel coated samples.

\subsection{Fire Performance of the Composites}

The flame spread tests were performed as screening tests, which then allow the selection of the coatings further used for the composite preparation. Composites were prepared using fabrics treated with A-T, B-T, B-TD, and B-TAlPi according to the results obtained from the flame spread test. Their FR performances were compared to the not treated reference using cone calorimeter test. The results of the cone calorimeter measurements are presented in Figure 14 and Table 3. The Figure 14 presents the different heat of release rate (HRR) curves obtained for each material, and Table 3 gives a mean value for each measurement using all the experiments performed for each composites, as well as the standard deviation associated with the measurements.

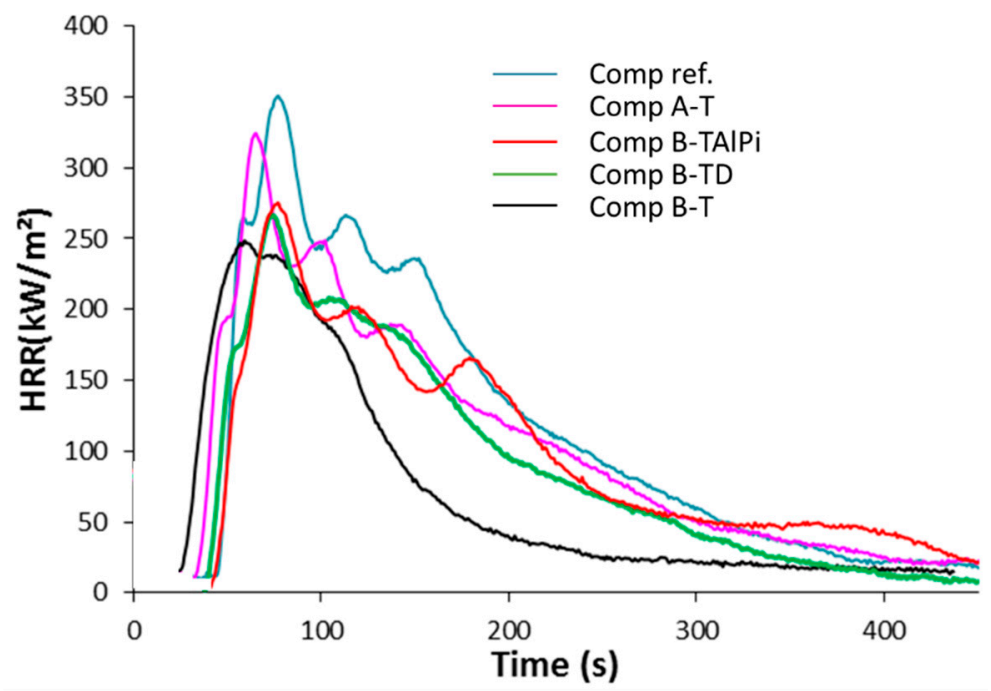

Figure 14. Heat release rate curves of the composites prepared with coated fabrics.

The sol-gel coatings of the flax fibers seem to be efficient enough to affect the global fire performances of the derived composites. A reduction of the $\mathrm{pHRR}$ is observed for all the samples compared to the untreated composite. For the composites made with fibers treated using only TEOS, it appears that the more concentrated the sol-gel is, the better the performances are in terms of pHRR and THR values. The effect is particularly significant on the THR, which decreases from 43 to $29 \mathrm{MJ}$. However the time to ignition is shortened. The comparison of the samples B-TAlPi, B-T, and B-TD 
shows that the addition of phosphorous did not further decrease the pHRR, as the values are similar for the composites prepared with the different fabrics. Moreover, slightly higher THR and time to ignition are observed when P-based additives or precursors are added to TEOS. Therefore, the benefit of using additional phosphorous in the coatings does not clearly appear. This phenomenon can be explained by the fact that the amount of phosphorus added to the fabrics by the coatings is low. Indeed, coatings add-on for these treatments is lower than 5 wt.- $\%$ but AlPi efficiency in PA11 generally appears at an additive level of 17-20 wt.\% [29]. Considering that the present composites have $45 \mathrm{wt} . \%$ matrix content, the amount of AlPi versus PA11 is too low to be efficient.

Table 3. Cone calorimeter results of the composites prepared with coated fabrics.

\begin{tabular}{ccccc}
\hline Sample Reference & Ti (s) & pHRR $\mathbf{( k W / \mathbf { m } ^ { 2 } )}$ & THR (MJ) & Residue (wt. $\%)$ \\
\hline Comp. ref. & $45 \pm 1$ & $349 \pm 2$ & $49 \pm 1$ & $36 \pm 1$ \\
Comp. A-T & $33 \pm 0$ & $286 \pm 20$ & $43 \pm 4$ & $39 \pm 2$ \\
Comp. B-T & $28 \pm 4$ & $244 \pm 6$ & $29 \pm 3$ & $49 \pm 0.4$ \\
Comp. B-TD & $36 \pm 4$ & $251 \pm 23$ & $34 \pm 7$ & $45 \pm 8$ \\
Comp. B-AlPi & $41 \pm 1$ & $250 \pm 35$ & $39 \pm 5$ & $44 \pm 4$ \\
\hline
\end{tabular}

\section{Conclusions}

The objective of this paper is the development of flame retardant PA11/Flax composites. The sol-gel approach was used to treat the fibers and provide the FR properties to the composite, as the literature shows good results to flame retard cellulosic fibers. Different formulation containing different concentrations of silicon precursors and additives were tested as well as different processing methodologies. The sol-gel coatings were first characterized using electronic microscopy (SEM and EPMA) and solid-state NMR. It was shown that, depending on the used precursors and protocol of deposition, the coatings can show two different aspects: a homogeneous coating all along the fibers or aggregates. The use of pure TEOS mainly leads to Q structures whereas APTES or DEPTES leads to $\mathrm{T}$ structures. The fire retardant properties of the sol-gel coated fibers and fabrics were improved, as well as those of the composites using such modified flax textiles. The best results are obtained when a high concentration of silicon-containing precursors is used. It was also demonstrated that the addition of phosphorus does not sharply improved the FR properties of the composites, because the sol-gel coatings do not contain enough of the phosphorous compound to be efficient.

Supplementary Materials: The following are available online at http://www.mdpi.com/2079-6439/7/10/86/s1, Figure S1. EDX analyses performed on the surface of the A-T fiber (Figure 2, SEM image of A-T, Points 1, 2 and 3), Figure S2. EDX analyses performed on the surface of the A-TD fiber (Figure 2, SEM image of A-TD, Points $1,2,3$, and 4), Figure S3. EDX analyses performed on the surface of the A-TDA fiber (Figure 2, SEM image of A-TDA, Point 1), Figure S4. EDX analyses performed on the surface of the A-TDA fiber (Figure 8, SEM image of A-T/TD/TA, Points 1, 2, 3, and 4), Figure S5. EDX analyses performed on the surface of the A-TAlPi fiber (Figure 10, SEM image of A-T/TD/TA, Points 1, 2 and 3), Figure S6. EDX analyses performed on the surface of the A-TAlPiMPP fiber (Figure 10, SEM image of A-T/TD/TA, Points 1, 2, and 3), Figure S7. Schematic representation of $\mathrm{D}, \mathrm{Ti}$, and Qi silicon structures.

Author Contributions: Conceptualization, F.S. and S.D.; Methodology, F.S. and S.D.; Investigation, S.B. and M.V.; Writing-Original Draft Preparation, F.S.; Writing-Review \& Editing, S.D.

Funding: This research received no external funding.

Acknowledgments: The authors would like to thank Chanel Leleux, Julien Molina, Julien Mahiques, Lison Podevin, and Ying Yang, Master's students at ENSCL, who have largely contributed to the experimental part of this work.

Conflicts of Interest: The authors declare no conflicts of interest. 


\section{References}

1. Lebaupin, Y.; Chauvin, M.; Hoang, T.-Q.T.; Touchard, F.; Beigbeder, A. Influence of constituents and process parameters on mechanical properties of flax fibre-reinforced polyamide 11 composite. J. Thermoplast. Compos. Mater. 2017, 30, 1503-1521. [CrossRef]

2. Gourier, C.; Bourmaud, A.; le Duigou, A.; Baley, C. Influence of PA11 and PP thermoplastic polymers on recycling stability of unidirectional flax fibre reinforced biocomposites. Polym. Degrad. Stab. 2017, 136, 1-9. [CrossRef]

3. Bourmaud, A.; le Duigou, A.; Gourier, C.; Baley, C. Influence of processing temperature on mechanical performance of unidirectional polyamide 11-flax fibre composites. Ind. Crop. Prod. 2016, 84, 151-165. [CrossRef]

4. Lebaupin, Y.; Chauvin, M.; Truong Hoang, T.Q.; Touchard, F. Effect of stacking sequence on low velocity impact and post-impact behavior of flax/polyamide 11 composites. In Proceedings of the ECCM 2016-17th European Conference on Composite Materials, Munich, Germany, 26-30 June 2016.

5. Kandola, B.K.; Horrocks, A.R.; Myler, P.; Blair, D. New developments in flame retardancy of glass-reinforced epoxy composites. J. Appl. Polym. Sci. 2003, 88, 2511-2521. [CrossRef]

6. Bourbigot, S.; Bachelet, P.; Samyn, F.; Jimenez, M.; Duquesne, S. Intumescence as method for providing fire resistance to structural composites: Application to poly(ethylene terephtalate) foam sandwich-structured composite. Compos. Interface 2013, 20, 269-277. [CrossRef]

7. Horrocks, A.R.; Kandola, B.K. Chapter 11. Flame resistant composites and nanocomposites. In Handbook of Fire Resistant Textiles, 1st ed.; Selcen Kilinc, F., Ed.; Woodhead Publishing: Cambridge, UK, 2013; pp. $283-321$.

8. Samyn, F.; Duquesne, S.; Bourbigot, S.; Ouagne, P. Finding the right balance between fire performances and mechanical properties. In Proceedings of the 25th Annual Conference on Recent Advances in Flame Retardancy of Polymeric Materials, Stamford, CT, USA, 19-21 May 2014.

9. Kandare, E.; Chukwunonso, A.K.; Kandola, B.K. The effect of fire-retardant additives and a surface insulative fabric on fire performance and mechanical property retention of polyester composites. Fire Mater. 2011, 35, 143-155. [CrossRef]

10. Chapple, S.; Anandjiwala, R. Flammability of natural fiber-reinforced composites and strategies for fire retardancy: A review. J. Thermoplast. Compos. Mater. 2010, 23, 871-893. [CrossRef]

11. Samyn, F.; Duquesne, S.; Bourbigot, S.; Ouagne, P. Strategies for flame retarding bio-composites. In Proceedings of the 8th Asian-Australasian Conference on Composite Materials ACCM 2012-Composites: Enabling Tomorrow's Industry Today, Kuala Lumpur, Malaysia, 6-8 November 2012; Jaafar, M., Azura, A.R., Leong, K.H., Leong, A.Y., Eds.; AACM: Phoenix, AZ, USA, 2012; pp. 155-160.

12. Duquesne, S.; Samyn, F.; Ouagne, P.; Bourbigot, S. Flame retardancy and mechanical properties of flax reinforced woven for composite applications. J. Ind. Text. 2015, 44, 665-681. [CrossRef]

13. Kandola, B.K.; Pornwannachai, W.; Alongi, J.; Caminade, E. Thermal protection of natural fibre thermoplastic composites using sol-gel coatings. In Proceedings of the Fire and Materials 2015-14th International Conference and Exhibition, San Francisco, CA, USA, 2-4 February 2015; pp. 453-463.

14. Bourbigot, S. Flame retardancy of textiles: New approaches. In Advances in Fire Retardant Materials, 1st ed.; Horrock, A.R., Price, D., Eds.; Woodhead publishing Ltd.: Cambridge, UK, 2008; pp. 9-40.

15. Horrocks, A.R.; Price, D. Fire Retardant Materials; Woodhead Publishing Ltd.: Cambridge, UK, 2001; pp. 128-181.

16. Bajaj, P. Handbook of Technical Textiles; Woodhead Publishing Ltd.: Cambridge, UK, 2000; pp. $223-263$.

17. Alongi, J.; Malucelli, G. State of the art and perspectives on sol-gel derived hybrid architectures for flame retardancy of textiles. J. Mater. Chem. 2012, 22, 21805-21809. [CrossRef]

18. Malucelli, G. Sol-gel flame retardant and/or antimicrobial finishings for cellulosic textiles. In Handbook of Renewable Materials for Coloration and Finishing; Mohd, Y., Ed.; Wiley: Hoboken, NJ, USA, 2018; pp. 501-519.

19. Castellano, A.; Colleoni, C.; Iacono, G.; Mezz, A.; Plutino, M.R.; Malucelli, G.; Rosace, G. Synthesis and characterization of a phosphorous/nitrogen based sol-gel coating as a novel halogen- and formaldehyde-free flame retardant finishing for cotton fabric. Polym. Degrad. Stab. 2019, 162, 148-159. [CrossRef]

20. Rosace, G.; Castellano, A.; Trovato, V.; Iacono, G.; Malucelli, G. Thermal and flame retardant behaviour of cotton fabrics treated with a novel nitrogen-containing carboxyl-functionalized organophosphorus system. Carbohydr. Polym. 2018, 196, 348-358. [CrossRef] [PubMed] 
21. Alongi, J.; Colleoni, C.; Rosace, G.; Malucelli, G. Phosphorus- and nitrogen-doped silica coatings for enhancing the flame retardancy of cotton: Synergisms or additive effects? Polym. Degrad. Stab. 2013, 98, 579-589. [CrossRef]

22. Bounor-Legaré, V.; Cassagnau, P. In situ synthesis of organic-inorganic hybrids or nanocomposites from sol-gel chemistry in molten polymers. Prog. Polym. Sci. 2014, 39, 1473-1497. [CrossRef]

23. Cardenas, A.; Hovnanian, N.; Smaihi, M. Sol-gel formation of heteropolysiloxanes from diethylphosphatoethy ltriethoxysilane and tetraethoxysilane. J. Appl. Polym. Sci. 1996, 60, 2279-2288. [CrossRef]

24. Hendrix, E.; Bostic, J.E.; Olson, J.S.; Barker, E.H. Pyrolysis combustion of cellulose, I. Effects of triphenyl phosphate in the presence of nitrogenous bases. J. Appl. Polym. Sci. 1970, 14, 1701-1723. [CrossRef]

25. Boryniec, S.; Przygocki, W. Polymer combustion processes. 3. Flame retardants for polymeric materials. Prog. Rubber Plast. Technol. 2001, 17, 59-80.

26. Bellayer, S.; Jimenez, M.; Gardelle, B.; Delaplace, G.; Bouquerel, J.; Duquesne, S.; Bourbigot, S. The electron microanalyzer (EPMA): A powerful device for the microanalysis of filled polymeric materials. Polym. Adv. Technol. 2015, 26, 1020-1026. [CrossRef]

27. Glaser, R.H.; Wilkes, G.L.; Bronnimann, C.E. Solid-state 29Si NMr of TEOS-based multifunctional sol-gel materials. J. Non-Cryst. Solids 1989, 113, 73-87. [CrossRef]

28. Garnier, A.; Da Cruz-Boisson, F.; Rigolet, S.; Brendlé, J.; Bounor-Legaré, V. Hydrolysis-condensation reactions of diethylphosphato-ethyltriethoxysilane involved in organic-inorganic talc-like hybrid synthesis: Liquid and solid-state NMR investigations. RSC Adv. 2016, 6, 75715-75723. [CrossRef]

29. Zhang, S.; Tang, W.; Gu, X.; Jiang, P.; Sun, J.; Duquesne, S.; Bourbigot, S.; Casetta, M. Flame retardancy and thermal and mechanical performance of intercalated, layered double hydroxide composites of polyamide 11, aluminum phosphinate, and sulfamic acid. J. Appl. Polym. Sci. 2016, 133, 43370. [CrossRef]

(C) 2019 by the authors. Licensee MDPI, Basel, Switzerland. This article is an open access article distributed under the terms and conditions of the Creative Commons Attribution (CC BY) license (http://creativecommons.org/licenses/by/4.0/). 